\title{
Microfiber Knot With Taper Interferometer for Temperature and Refractive Index Discrimination
}

\author{
André D. Gomes and Orlando Frazão
}

\begin{abstract}
A compact sensing structure using two distinct optical devices, a microfiber knot resonator and an abrupt taper-based Mach-Zehnder interferometer (MZI), is presented. The device was fabricated using only $\mathrm{CO}_{2}$ laser processing. The transmission spectrum presents two different components with different sensitivities to different physical and chemical parameters. The sensor was characterized in temperature and refractive index. For temperature sensing in water, the MZI component presents a sensitivity of $-196 \pm 2 \mathrm{pm} /{ }^{\circ} \mathrm{C}$ while the microfiber knot resonator (MKR) component shows a sensitivity of $25.1 \pm 0.9 \mathrm{pm} /{ }^{\circ} \mathrm{C}$, for water temperature variations of $12{ }^{\circ} \mathrm{C}$. Sensitivities of $1354 \pm 14 \mathrm{~nm} / \mathrm{RIU}$ and $-43 \pm 4 \mathrm{~nm} / \mathrm{RIU}$ were achieved for refractive index sensing for the MZI and the MKR components, respectively, in a refractive index range from 1.32823 to 1.33001 . The matrix method was used for the simultaneous measurement of temperature and refractive index.
\end{abstract}

Index Terms-Optical fiber sensor, optical interferometry, microfiber knot resonator, temperature measurement, refractive index, salinity.

\section{INTRODUCTION}

$\mathbf{M}$ ICROFIBER knot resonators (MKR) have been widely studied as sensing elements in the past few years. This type of device had a significant impact in the field of sensing and also in other fields, such as ultrafast optics. The taper in the knotted zone allows the evanescent field of light to couple between the adjacent sections of the taper creating a resonance [1]. Monitoring the sensor resonance, a wide variety of parameters were able to be measured, such as temperature [2]-[4], concentration of sodium chloride and refractive index (RI) [5]-[7], among others. For refractive index sensing, a simple MKR in a Sagnac loop reflector was reported [5] with a sensitivity of $30.49 \mathrm{~nm} / \mathrm{RIU}$ in a range from 1.334-1.348. For the same purpose, a Teflon-coated MKR was demonstrated with a sensitivity of $30.5 \mathrm{~nm} / \mathrm{RIU}$ in a range from 1.3322 to 1.3412 . High sensitivity can be achieved by using different configurations, such as cascaded MKRs presented by Xu et al. [7]. This device achieved a sensitivity of $6523 \mathrm{~nm} / \mathrm{RIU}$ in a refractive index range from 1.3320 to 1.3350. Apart from MKRs, other resonating structures were

Manuscript received May 16, 2017; revised July 10, 2017; accepted July 31, 2017. Date of publication August 2, 2017; date of current version August 14, 2017. This work was supported by the Project NanoSTIMANorth Portugal Regional Operational Programme under Grant NORTE-010145-FEDER-000016. The work of A. Gomes was supported by FCT under Grant PD/BI/129000/2017. (Corresponding author: André D. Gomes.)

The authors are with INESC TEC, 4169-008 Porto, Portugal, and also with the Department of Physics and Astronomy, Faculty of Sciences, University of Porto, 4169-007 Porto, Portugal (e-mail: ardcgomes@gmail.com; ofrazao@inescporto.pt).

Color versions of one or more of the figures in this letter are available online at http://ieeexplore.iee.org.

Digital Object Identifier 10.1109/LPT.2017.2735185

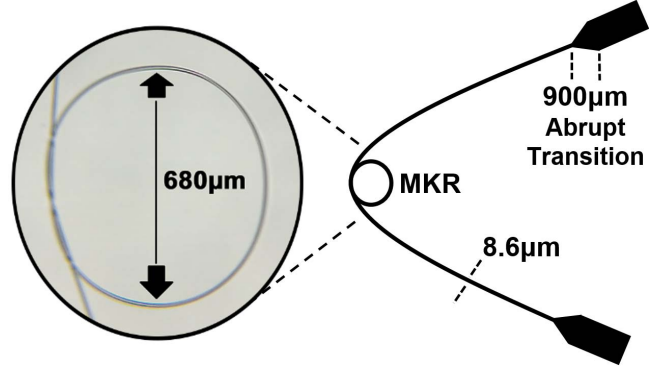

Fig. 1. Schematic of the proposed sensor and picture of the microfiber knot resonator.

also demonstrated to be good candidates for refractive index sensing, such as microspheres and microbubbles [8], [9].

The Mach-Zehnder interferometer (MZI) technique is mainly used for measuring the refractive index of a medium due to the induced phase shift of the guided light created by the analyzed medium [10]. However, year after year new configurations were developed increasing, therefore, the scope of this sensor. Different optical fiber MZI configurations have been reported for refractive index [11]-[13] and temperature sensing. For refractive index, a simple biconical fiber taper [11] can achieve sensitivities of around $705 \mathrm{~nm} / \mathrm{RIU}$ in a range of 1.333 to 1.350 . Higher sensitivities can be obtained by creating a taper-based MZI in a thinned optical fiber [12]. Such sensor can achieve a maximum sensitivity of $2210.84 \mathrm{~nm} / \mathrm{RIU}$ between 1.3997 and 1.4096. Different configurations, such as an MZI based on a large knot fiber resonator can also be used for refractive index sensing [13], presenting a sensitivity of $642 \mathrm{~nm} / \mathrm{RIU}$ in a wide range of refractive indices (1.3735 to 1.428 ).

Recently, several configurations using MZIs have been demonstrated for simultaneous measurement of temperature and refractive index [14]-[18]. In the last year, a microfiber MZI with a knot resonator was presented for the same purpose [19], [20].

In this Letter, a different approach is presented for simultaneous measurement of temperature and refractive index using the combination of two different optical structures: a microfiber knot resonator integrated in a Mach-Zehnder interferometer based on an abrupt fiber taper. This compact sensor is fabricated using the same technology $-\mathrm{CO}_{2}$ laser processing.

\section{Experimental Setup AND Results}

Figure 1 presents a schematic of the sensor. A microfiber knot resonator with a diameter of around $680 \mu \mathrm{m}$ was created using a $50 \mathrm{~mm}$-long abrupt fiber taper. $\mathrm{A} \mathrm{CO}_{2}$ laser system 


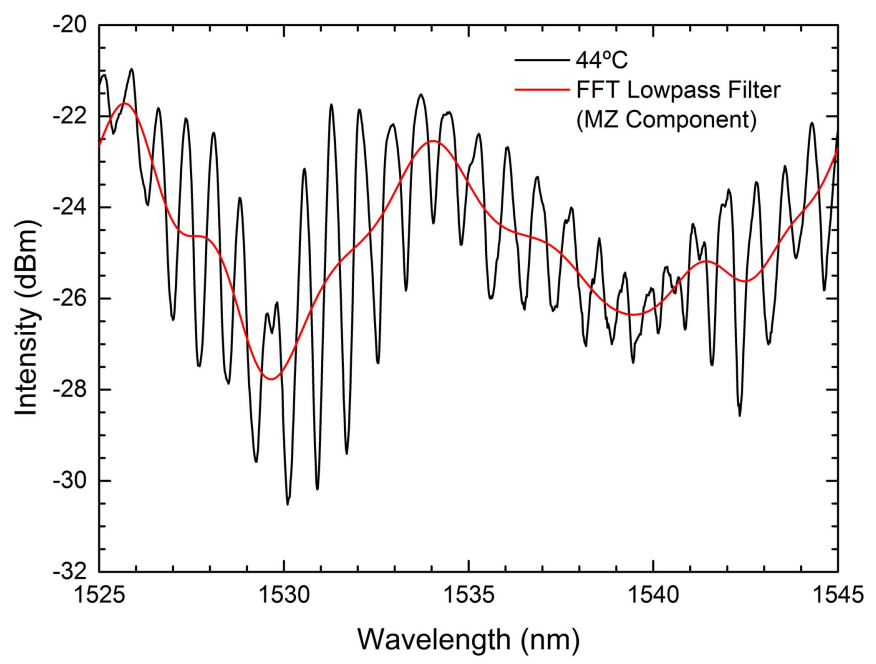

Fig. 2. Sensor transmission spectrum in water at $44{ }^{\circ} \mathrm{C}$ and filtered MZI spectral component (lowpass filter cutoff frequency: $0.5 \mathrm{~nm}^{-1}$ ).
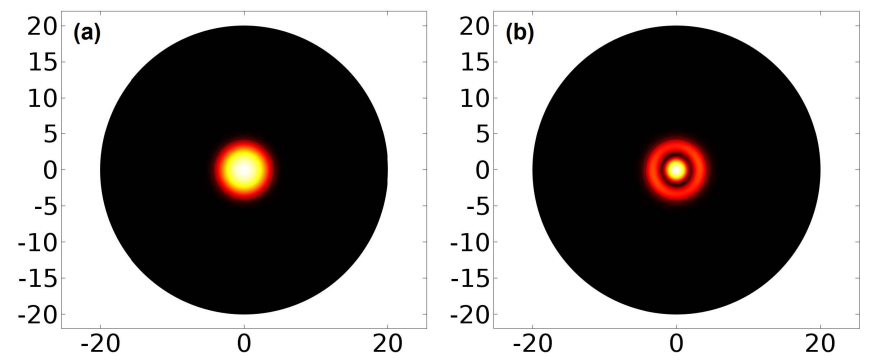

Fig. 3. Simulated mode profiles for the first two modes: (a) $\mathrm{LP}_{01}$ : effective refractive index of 1.4385 (b) $\mathrm{LP}_{02}$ : effective refractive index of 1.4149 .

was used to produce the abrupt taper with a waist diameter of $8.6 \mu \mathrm{m}$. The abrupt transition regions are $900 \mu \mathrm{m}$-long, allowing the excitation of modes with different effective refractive indices in the beginning of the taper, which will then recouple in the knot region due to the inherent curvature of the structure, creating an MZI.

The sensor was connected between a broadband optical source and an optical spectrum analyzer (OSA). The optical source had a central wavelength of $1550 \mathrm{~nm}$ and a bandwidth of $100 \mathrm{~nm}$. Transmission measurements were performed with a resolution of $0.04 \mathrm{~nm}$. Figure 2 shows transmission spectrum of the sensor immersed in water at $44{ }^{\circ} \mathrm{C}$. The transmission spectrum is the combination of the MZI with the MKR response. Since the MKR resonant frequency is higher than the MZI interference pattern, an FFT lowpass filter can easily remove the MKR resonance allowing to measure the MZI component.

The MZI is caused by the effective refractive index difference between the different modes excited by the abrupt taper, mainly the fundamental and the second order mode. The effective refractive indices of the first modes $\left(\mathrm{LP}_{01}\right.$ and $\left.\mathrm{LP}_{02}\right)$ were calculated using COMSOL Multiphysics. The external medium refractive index used for the simulations was 1.32917 (water at $44{ }^{\circ} \mathrm{C}$ ) and the fiber refractive index was 1.444 (refractive index of silica). The simulated refractive indices for the first 2 modes of higher power were 1.4385 and 1.4149 , respectively. The mode profiles are depicted in Figure 3.
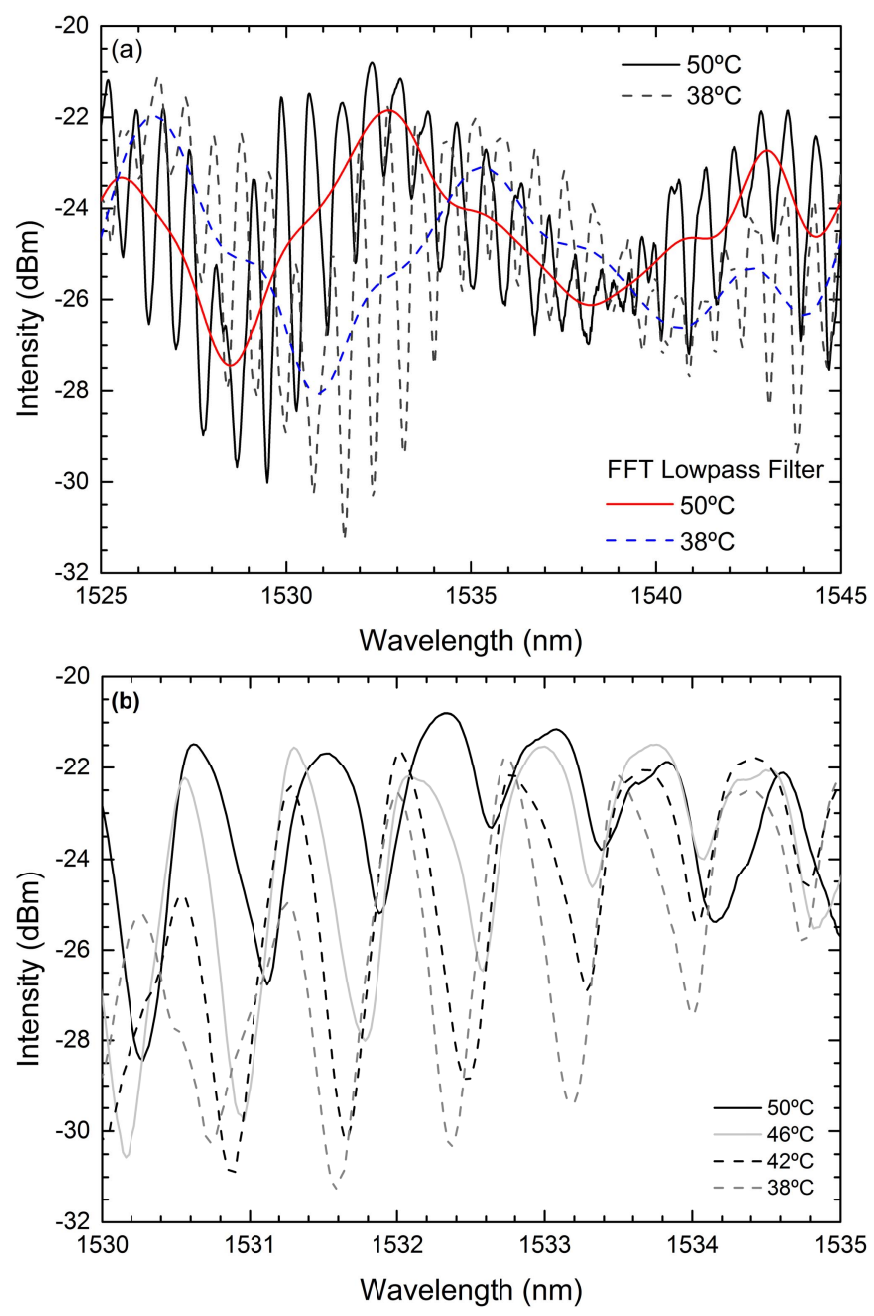

Fig. 4. Transmission spectra in water at different temperatures: (a) Full spectra (MZI and MKR) and filtered MZI spectral components (lowpass filter cutoff frequency: $0.5 \mathrm{~nm}^{-1}$. (b) Microfiber knot resonator component.

The MZI physical length is given by:

$$
L=\frac{\lambda_{1} \lambda_{2}}{\Delta n \times F S R}
$$

where $\lambda_{1}$ and $\lambda_{2}$ are the wavelengths of two consecutive interference peaks, $\Delta n$ is the effective refractive index difference between the first 2 modes, and FSR is the free spectral range. From the filtered MZI component in Figure 2, the first interference peaks are located at $1528.86 \mathrm{~nm}$ and $1538.48 \mathrm{~nm}$, which corresponds to a free spectral range of $9.62 \mathrm{~nm}$. Hence, using (1) the physical length of the MZI is $10.4 \mathrm{~mm}$, which matches the distance between the beginning of the taper and the knot region (the knot is not centered in the middle of the taper).

For the MKR, considering the two consecutive resonant wavelengths $1530.12 \mathrm{~nm}$ and $1530.90 \mathrm{~nm}$, one can calculate the knot diameter from the expression:

$$
d=\frac{\lambda_{1} \lambda_{2}}{\pi \times n_{e f f} \times F S R}
$$

Adopting the simulated effective refractive index for the fundamental mode, the calculated knot diameter is $664 \mu \mathrm{m}$. 

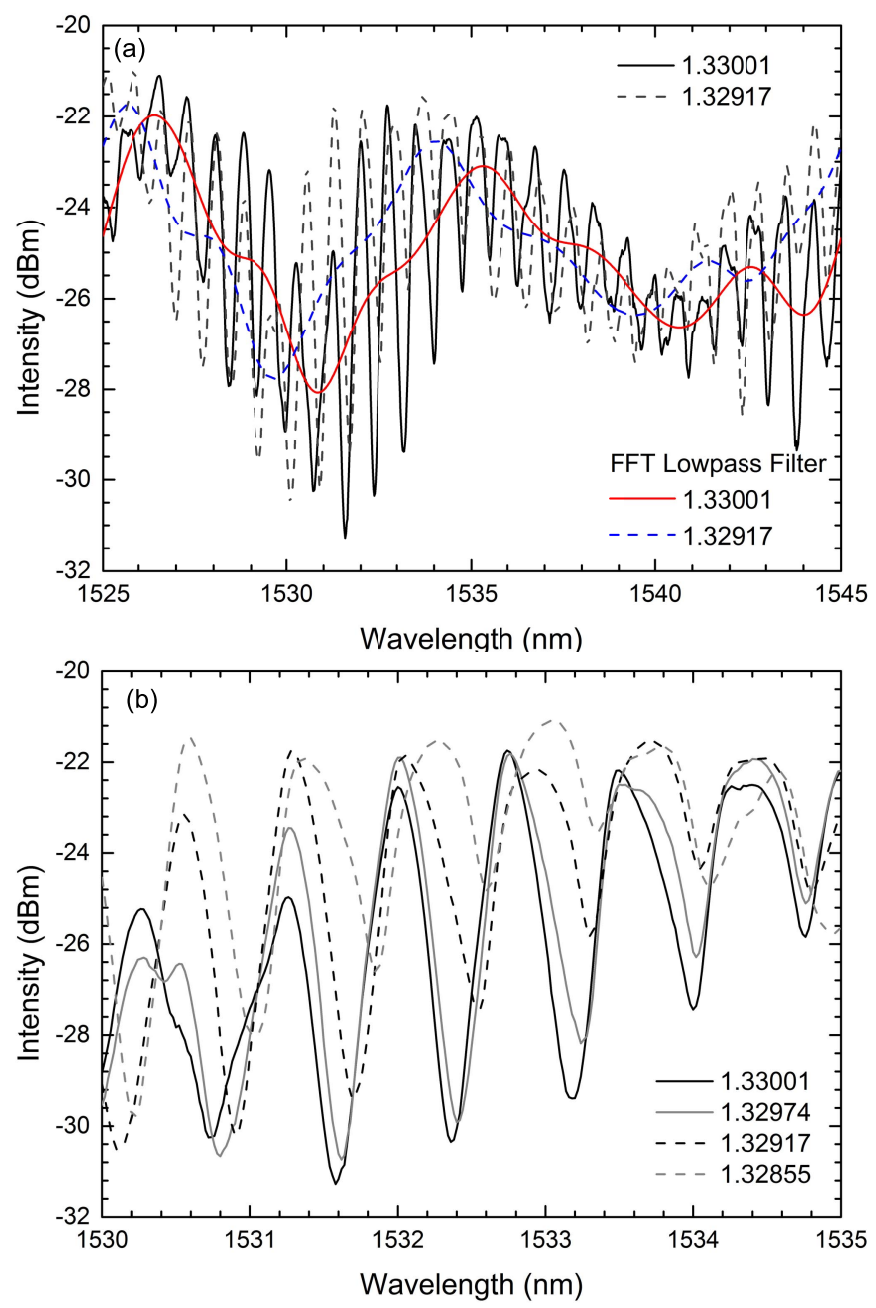

Fig. 5. Transmission spectra in water at 2 different refractive indices: (a) Full spectra (MZI and MKR) and filtered MZI spectral components (lowpass filter cutoff frequency: $0.5 \mathrm{~nm}^{-1}$. (b) Microfiber knot resonator component.

The MKR response occurs for both modes and they present similar FSR and peak positions. However, $\mathrm{LP}_{01}$ is dominant and the resonance caused by $\mathrm{LP}_{02}$ has lower visibility. Its presence is still visible in Figure 2, near $1540 \mathrm{~nm}$.

Temperature measurements were performed by immersing the sensor in water while progressively decreasing the temperature from $50{ }^{\circ} \mathrm{C}$ to $38{ }^{\circ} \mathrm{C}$. Figure 4 (a) presents the transmission spectra at two different temperatures. The MZI peak red shifts with the decrease of temperature [Fig. 4(a)] while the MKR peak blue shifts, as observed in Figure 4(b).

In terms of refractive index, a temperature-RI conversion scale was used based on the RI dependence of water with temperature, constant pressure, and wavelength [21]. The conversion of water temperature in degrees Celsius to RI is given by:

$$
\begin{aligned}
n(T)=1.33315- & 3.14566 \times 10^{-5} T \\
- & 1.35711 \times 10^{-6} T^{2}+3.626 \times 10^{-10} T^{3}
\end{aligned}
$$

From these values the sensor response to refractive index variations was obtained.

Figure 5(a) presents the transmission spectra of the sensor under two different refractive indices. In this case,
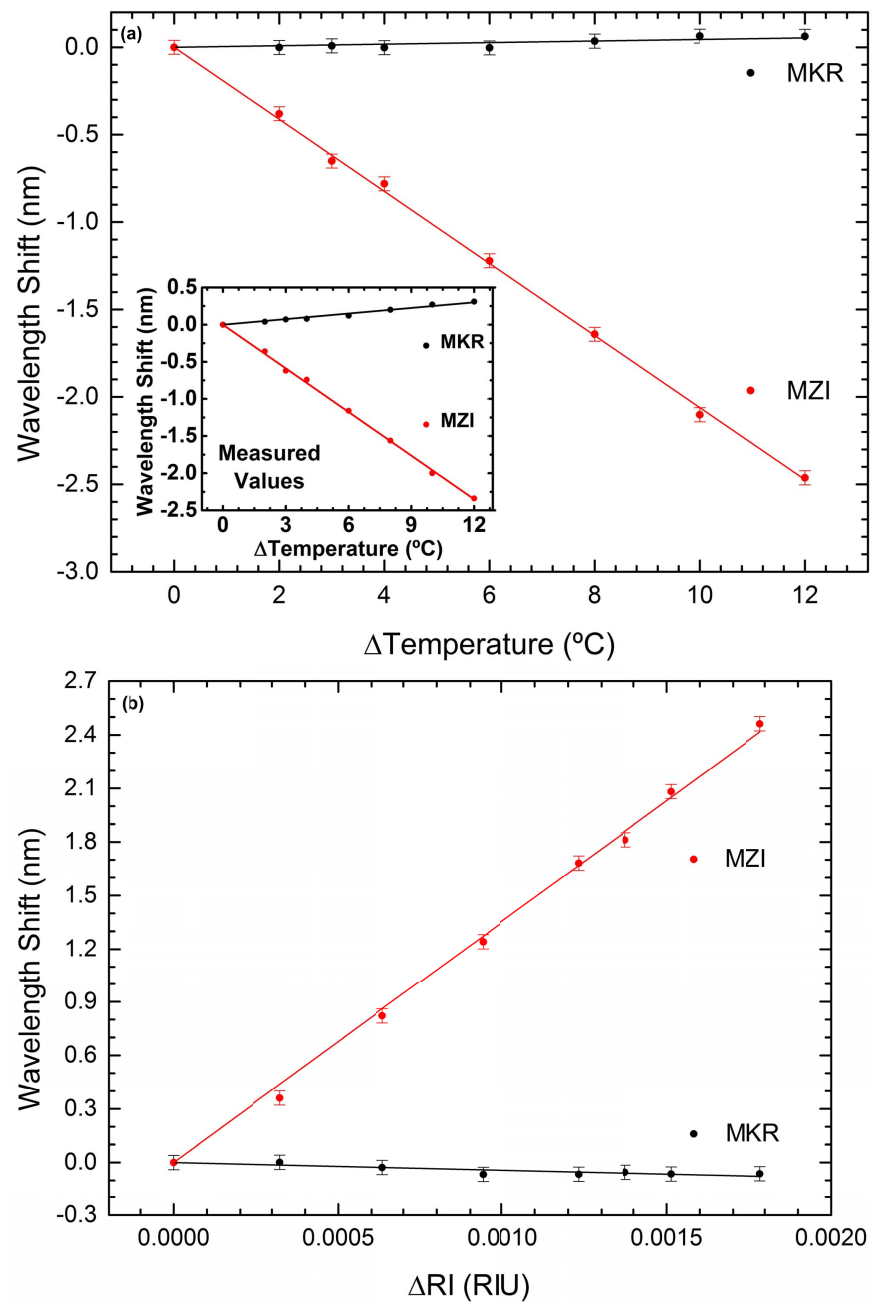

Fig. 6. (a) Wavelength shift as a function of water temperature variation for both components (MKR and MZI) after removing the temperature sensitivity in air. The inner plot shows the measured values before extracting the temperature behavior in air. (b) Wavelength shift as a function of refractive index variation for both components (MKR and MZI).

the MZI peak blue shifts with the decrease of the external refractive index while the MKR peak red shifts, as seen in Figure 5(b).

The wavelength shift as a function of the temperature variation in water for both components, the MZI and the MKR, is depicted in the inner plot of Figure 6(a). The MZI interference peak around $1530 \mathrm{~nm}$ and the resonance peak around $1531.7 \mathrm{~nm}$ were used for the sensor characterization. In water, temperature sensitivities of $-196 \pm 2 \mathrm{pm} /{ }^{\circ} \mathrm{C}$ and $25.1 \pm 0.9 \mathrm{pm} /{ }^{\circ} \mathrm{C}$ were obtained for the MZI and the MKR, respectively. Such values correspond to the effect of temperature variation but also to the effect of water refractive index variation due to thermo-optic effects. In order to obtain a correct response of the sensor to water refractive index variations, one need to remove the temperature behavior in air [22]. The temperature sensitivity of the structure in air is $10.2 \mathrm{pm} /{ }^{\circ} \mathrm{C}$ and $20.6 \mathrm{pm} /{ }^{\circ} \mathrm{C}$, respectively for the MZI and MKR. Removing this component the sensor response due to refractive index variations is obtained, whose result is depicted in Figure 6(a). The temperature values need to be 
converted to the correspondent refractive indices values using equation 3. Applying this, the wavelength shift as a function of the water refractive index variation for both components is shown in Figure 6(b). The obtained refractive index sensitivity was $1354 \pm 14 \mathrm{~nm} / \mathrm{RIU}$ for the MZI and $-43 \pm 4 \mathrm{~nm} / \mathrm{RIU}$ for the MKR. The refractive index ranged from 1.32823 to 1.33001 .

The difference in the sensitivity coefficients allows the possibility of using the sensor for simultaneous measurement of temperature and refractive index. The matrix of relation between refractive index and temperature variations, and wavelength shifts can be obtained using the sensitivities of each component to the measured parameters [23]. With this, the matrix expression for refractive index and temperature variations as a function of the sensitivities and wavelength shifts is:

$$
\left[\begin{array}{c}
\Delta n \\
\Delta T
\end{array}\right]=\frac{1}{D}\left[\begin{array}{cc}
k_{2 T} & -k_{1 T} \\
-k_{2 n} & k_{1 n}
\end{array}\right]\left[\begin{array}{c}
\Delta \lambda_{M Z I} \\
\Delta \lambda_{M K R}
\end{array}\right]
$$

where $D=k_{1 n} k_{2 T}-k_{2 n} k_{1 T}$ is the determinant of the relationship matrix, being $k_{i n}$ and $k_{i T}$ the sensitivities to refractive index and temperature.

Using the previously calculated sensitivities, the matrix can be expressed as:

$$
\left[\begin{array}{c}
\Delta n \\
\Delta T
\end{array}\right]=0.0353\left[\begin{array}{cc}
0.0206 & -0.0102 \\
43 & 1354
\end{array}\right]\left[\begin{array}{c}
\Delta \lambda_{M Z I} \\
\Delta \lambda_{M K R}
\end{array}\right]
$$

where $\Delta \lambda_{M Z I}$ and $\Delta \lambda_{M K R}$ are in nanometers and the obtained refractive index and temperature variations are in refractive index units and degrees Celsius, respectively.

\section{CONCLUSION}

In summary, a new compact sensing structure using two distinct optical devices, a microfiber knot resonator and an abrupt taper-based Mach-Zehnder interferometer, was fabricated using only $\mathrm{CO}_{2}$ laser processing. The sensor presents two different spectral components that respond differently to each measured parameter. A characterization in temperature and refractive index was performed. In terms of temperature, the MZI component presents a sensitivity of $-196 \pm 2 \mathrm{pm} /{ }^{\circ} \mathrm{C}$ while the MKR component shows a sensitivity of $25.1 \pm 0.9 \mathrm{pm} /{ }^{\circ} \mathrm{C}$, for water temperature variations of $12{ }^{\circ} \mathrm{C}$. A temperature sensitivity in air of $10.2 \mathrm{pm} /{ }^{\circ} \mathrm{C}$ and $20.6 \mathrm{pm} /{ }^{\circ} \mathrm{C}$ were obtained for the $\mathrm{MZI}$ and the MKR, respectively. The sensor response to water refractive index variation was achieved by removing the temperature response of the structure in air to the temperature response in water [22]. Sensitivities of $1354 \pm 14 \mathrm{~nm} / \mathrm{RIU}$ and $-43 \pm 4 \mathrm{~nm} / \mathrm{RIU}$ were achieved for refractive index sensing for the MZI and the MKR components, respectively, in a water refractive index range from 1.32823 to 1.33001 . The device can be used for simultaneous measurement of temperature and refractive index using the relationship matrix between the different measured parameters. The proposed sensor is more compact and stable than similar reported structures [19], [20], since the MZI relies on a single taper structure and not on a second fiber tip connected through Van der Waals forces. Structures like [19] and [20] which use coupling between two microfibers can be very fragile and unstable.

\section{REFERENCES}

[1] X. Jiang et al., "Demonstration of optical microfiber knot resonators," Appl. Phys. Lett., vol. 88, no. 22, p. 223501, 2006.

[2] X. Zeng, Y. Wu, C. Hou, J. Bai, and G. Yang, "A temperature sensor based on optical microfiber knot resonator," Opt. Commun, vol. 282, no. 18, pp. 3817-3819, 2009.

[3] H. Yang, S. Wang, X. Wang, Y. Liao, and J. Wang, "Temperature sensing in seawater based on microfiber knot resonator," Sensors, vol. 14, no. 10 , pp. 18515-18525, 2014.

[4] Y. Wu, Y.-J. Rao, Y.-H. Chen, and Y. Gong, "Miniature fiber-optic temperature sensors based on silica/polymer microfiber knot resonators," Opt. Exp., vol. 17, no. 20, pp. 18142-18147, 2009.

[5] K.-S. Lim, I. Aryanfar, W.-Y. Chong, Y.-K. Cheong, S. W. Harun, and $\mathrm{H}$. Ahmad, "Integrated microfibre device for refractive index and temperature sensing," Sensors, vol. 12, no. 9, pp. 11782-11789, 2012.

[6] X. Li and H. Ding, "A stable evanescent field-based microfiber knot resonator refractive index sensor," IEEE Photon. Technol. Lett., vol. 26, no. 16, pp. 1625-1628, Aug. 15, 2014.

[7] Z. Xu et al., "Highly sensitive refractive index sensor based on cascaded microfiber knots with Vernier effect," Opt. Exp., vol. 23, no. 5, pp. 6662-6672, 2015.

[8] P. Wang et al., "Lead silicate glass microsphere resonators with absorption-limited Q," Appl. Phys. Lett., vol. 98, no. 18, p. 181105, 2011.

[9] P. Wang et al., "Lead-silicate glass optical microbubble resonator," Appl. Phys. Lett., vol. 106, no. 6, p. 061101, 2015.

[10] T. Schubert, N. Haase, R. Gottfried-Gottfried, and H. Kück, "Refractiveindex measurements using an integrated Mach-Zehnder interferometer," Sens. Actuators A, Phys., vol. 60, nos. 1-3, pp. 108-112, 1997.

[11] K. Q. Kieu and M. Mansuripur, "Biconical fiber taper sensors," IEEE Photon. Technol. Lett., vol. 18, no. 21, pp. 2239-2241, Nov. 1, 2006.

[12] J. Yang et al., "High sensitivity of taper-based Mach-Zehnder interferometer embedded in a thinned optical fiber for refractive index sensing," Appl. Opt., vol. 50, no. 28, pp. 5503-5507, 2011.

[13] A. D. Gomes and O. Frazão, "Mach-Zehnder based on large knot fiber resonator for refractive index measurement," IEEE Photon. Technol. Lett., vol. 28, no. 12, pp. 1279-1281, Jun. 15, 2016.

[14] L. Li, L. Xia, Z. Xie, L. Hao, B. Shuai, and D. Liu, "In-line fiber Mach-Zehnder interferometer for simultaneous measurement of refractive index and temperature based on thinned fiber," Sens. Actuators A, Phys., vol. 180, pp. 19-24, Jun. 2012.

[15] P. Lu, L. Men, K. Sooley, and Q. Chen, "Tapered fiber Mach-Zehnder interferometer for simultaneous measurement of refractive index and temperature," Appl. Phys. Lett., vol. 94, no. 13, p. 131110, 2009.

[16] Y. Cao, H. Liu, Z. Tong, S. Yuan, and J. Su, "Simultaneous measurement of temperature and refractive index based on a Mach-Zehnder interferometer cascaded with a fiber Bragg grating," Opt. Commun., vol. 342, pp. 180-183, May 2015.

[17] H. Luo, Q. Sun, Z. Xu, D. Liu, and L. Zhang, "Simultaneous measurement of refractive index and temperature using multimode microfiberbased dual Mach-Zehnder interferometer," Opt. Lett., vol. 39, no. 13, pp. 4049-4052, 2014.

[18] H. Luo, Q. Sun, Z. Xu, W. Jia, D. Liu, and L. Zhang, "Microfiber-based inline Mach-Zehnder interferometer for dual-parameter measurement," IEEE Photon. J., vol. 7, no. 2, Apr. 2015, Art. no. 7100908.

[19] Y. Liao, J. Wang, S. Wang, H. Yang, and X. Wang, "Simultaneous measurement of seawater temperature and salinity based on microfiber MZ interferometer with a knot resonator," J. Lightw. Technol., vol. 34 , no. 23, pp. 5378-5384, Dec. 1, 2016.

[20] Y. Liao, J. Wang, S. Wang, H. Yang, and X. Wang, "Spectral characteristics of the microfiber MZ interferometer with a knot resonator," Opt. Commun., vol. 389, pp. 253-257, Apr. 2017.

[21] E. W. Washburn, International Critical Tables of Numerical Data, Physics, Chemistry and Technology. New York, NY, USA: McGraw-Hill, 1930.

[22] R. M. André et al., "Simultaneous measurement of temperature and refractive index using focused ion beam milled Fabry-Perot cavities in optical fiber micro-tips," Opt. Exp., vol. 24, no. 13, pp. 14053-14065, 2016.

[23] F. Farahi, D. J. Webb, J. D. C. Jones, and D. A. Jackson, "Simultaneous measurement of temperature and strain: Cross-sensitivity considerations," J. Lightw. Technol., vol. 8, no. 2, pp. 138-142, Feb. 1990. 